\title{
HARMONICITY OF A FOLIATION AND OF AN ASSOCIATED MAP
}

\author{
Philippe Tondeur and Lieven Vanhecke
}

\begin{abstract}
A foliation on a Riemannian manifold $(M, g)$ is harmonic if all the leaves are minimal submanifolds. We give a new characterisation of the harmonicity of a foliation on $(M, g)$ by the harmonicity of an associated bundle map of $\left(T M, g^{C}\right)$, where $g^{C}$ is the complete lift metric of $g$ to the tangent bundle as introduced by Yano and Ishihara.
\end{abstract}

\section{INTRODUCTION AND STATEMENT OF THE RESULT}

A foliation $\mathcal{F}$ on a Riemannian manifold is harmonic if all the leaves of $\mathcal{F}$ are minimal submanifolds of $(M, g)$. The reason for this terminology (introduced in [7]) is that the local submersions defining $\mathcal{F}$ in distinguished charts are harmonic maps $[2,3,4]$ precisely when $\mathcal{F}$ is a harmonic foliation $[7$, Theorem 2.28 and Theorem 3.3, (i), (ii)]. A foliation $\mathcal{F}$ on a manifold $M$ is (geometrically) taut if a metric $g$ exists on $M$ which turns $\mathcal{F}$ into a harmonic foliation. There is a simple topological (cohomological) criterion characterising the tautness of a foliation $\mathcal{F}$. For simplicity, we assume throughout this note that the tangent bundle $L$ and the normal bundle $Q=T M / L$ of $\mathcal{F}$ are oriented (and hence also $M$ is oriented). The dimension of the leaves is denoted $p, 0<p<n=\operatorname{dim} M$.

Rummler - Sullivan Criterion for Tautness. $[12,13]$. Let $g_{L}$ be a Riemannian (fiber) metric on $L$ with volume form $\omega_{L}$ along the leaves. Then $\mathcal{F}$ is harmonic for a metric $g$ on $M$ restricting to $g_{L}$ on $L$ if and only if $\omega_{L}$ is the restriction of a $p$-form $\chi$ on $M$ satisfying

$$
d \chi\left(X_{1}, \cdots, X_{p+1}\right)=0
$$

if $p$ of the vector fields $X_{1}, \cdots, X_{p+1}$ are tangent to $\mathcal{F}$.

For $p=n-1$ this condition simply states that $\chi$ is a closed form. For Riemannian foliations $[11,14,15]$, this criterion takes a particularly simple form (see $[8,9,16])$.

Many examples of harmonic foliations were given in [7]. They include totally geodesic foliations, foliations of Kähler manifolds by complex submanifolds, codimension one foliations orthogonal to a divergence free unit vector field [7, Proposition 3.9],

Received 26th October, 1995.

This work was partially supported by NATO grant CRG.910034.

Copyright Clearance Centre, Inc. Serial-fee code: 0004-9729/96 \$A2.00+0.00. 
or equivalently, defined by the vanishing of a co-closed one-form of unit length, and Roussarie's foliation of $\Gamma \backslash S L(2, \mathbb{R})$ with $\Gamma \subset S L(2, \mathbb{R})$ discrete and cocompact [7, Proposition 3.34]. The last example is certainly not Riemannian since its GodbillonVey class is non-trivial.

In this note we give a new characterisation of the harmonicity of a foliation $\mathcal{F}$ on $(M, g)$. We shall recall the definition of the Gray-O'Neill tensor $T$ and the mean curvature form $\kappa$ of $\mathcal{F}$ in Section 2. Define a $(0,2)$-tensor field by

$$
\Phi(E, F)=\kappa\left(T_{E} F\right) .
$$

Let $\varphi: T M \rightarrow T M$ be the associated endomorphism field given by

$$
\Phi(E, F)=g(\varphi(E), F) .
$$

Consider the complete lift metric $g^{C}$ on $T M$ of Yano and Ishihara [17]. This is the semi-Riemannian metric of signature $(n, n)$ defined by

$$
\left\{\begin{array}{l}
g^{C}\left(X^{H}, Y^{H}\right)=g^{C}\left(X^{V}, Y^{V}\right)=0, \\
g^{C}\left(X^{H}, Y^{V}\right)=g^{C}\left(X^{V}, Y^{H}\right)=g(X, Y)^{V} .
\end{array}\right.
$$

Here the horizontal and vertical lifts of tangent vector fields $X, Y$ on $M$ refer to the decomposition of the tangent space of $T M$ at every point in horizontal vectors with respect to the Levi Civita connection $\nabla$ associated to $g$ and canonical vertical vectors. For vector fields $X, Y$ on $M$ the function $g(X, Y)^{V}$ on $T M$ is the pull-back of $g(X, Y)$ under the projection $T M \rightarrow M$.

With these definitions out of the way, we can state our main result as follows.

ThEOREM. Let $\mathcal{F}$ be a foliation on the Riemannian manifold $(M, g)$. Then $\mathcal{F}$ is harmonic if and only if the map $\varphi:\left(T M, g^{C}\right) \rightarrow\left(T M, g^{C}\right)$, viewed as a map of semi-Riemannian manifolds, is a harmonic map.

In fact, we shall prove moreover that if this map is harmonic, it necessarily reduces to the 0-map on each fiber, that is, $\varphi=\pi$, the projection $T M \rightarrow M$.

\section{Preliminaries}

Let $(M, g)$ be an $n$-dimensional (oriented) Riemannian manifold, and $g^{C}$ the complete lift of the metric $g$ to $T M$ as defined by (1.3). This metric can also be considered as the horizontal lift $g^{H}$ of $g$ when this is considered with respect to the Levi Civita connection $\nabla$ associated to $g[17]$. Note that by definition (1.3) horizontal and vertical lifts of vector fields on $M$ are null vectors for $g^{C}$.

Now, consider a $(1,1)$-tensor field on $M$ as a map $\varphi:\left(T M, g^{C}\right) \rightarrow\left(T M, g^{C}\right)$. The following characterisation of its harmonicity was given in [5]. 
PROPOSITION. $\varphi$ is harmonic if and only if $\nabla^{*} \varphi=0$, where $\nabla^{*}$ denotes the formal adjoint of $\nabla$.

$\nabla^{*} \varphi$ is the vector field defined as follows. In local coordinates with local components $\varphi_{i}^{k}$ and where $\left(g^{i j}\right)$ denotes the inverse matrix of $\left(g_{i j}\right)$, we have

$$
\left(\nabla^{*} \varphi\right)^{k}=-\sum_{i, j} g^{i j} \nabla_{i} \varphi_{j}^{k}
$$

Next, we recall the definition of the Gray-O'Neill tensor $T$ of a foliation $\mathcal{F}$ on $(M, g)[6,10]$. Let $\pi: T M \rightarrow L^{\perp}$ be the orthogonal projection to $L^{\perp} \cong Q$ with respect to $g$, and $\pi^{\perp}: T M \rightarrow L$ the orthogonal projection to $L$. Then

$$
T_{E} F=\pi\left(\nabla_{\left.\pi^{\perp} E \pi^{\perp} F\right)+\pi^{\perp}\left(\nabla_{\pi^{\perp} E} \pi\right)}\right.
$$

for vector fields $E, F$ on $M$. The conventions adapted below are $U, V, W \in \Gamma L$ and $X, Y, Z \in \Gamma L^{\perp}$. Clearly $T_{E}=T_{\pi^{\perp} E}$. Moreover, we have [6]

$$
\begin{aligned}
& T_{X} U=0, \quad T_{X} Y=0 \\
& T_{U} V=\pi\left(\nabla_{U} V\right), \quad T_{U} X=\pi^{\perp}\left(\nabla_{U} X\right) \\
& T_{U} V=T_{V} U ; \\
& T_{U} \quad \text { is alternating, in particular } g\left(T_{U} V, X\right)=-g\left(V, T_{U} X\right) .
\end{aligned}
$$

The mean curvature vector field or tension field $\tau$ of $\mathcal{F}$ on $(M, g)$ is given by $[14$, $6.16]$

$$
\tau=\sum_{i=1}^{p} T_{U_{i}} U_{i}=\sum_{i=1}^{p} \pi\left(\nabla_{U_{i}} U_{i}\right)
$$

for a (local) orthonormal frame $U_{1}, \cdots, U_{p}$ of $L$. Note that we have suppressed the usual factor $p^{-1}$. The mean curvature one-form $\kappa$ is defined by

$$
\kappa(E)=g(\tau, E)
$$

and satisfies $\kappa(U)=0$ for $U \in \Gamma L$. The harmonicity of $\mathcal{F}$ is characterised by $\tau=0$ or $\kappa=0$.

\section{Proof of the theorem}

We return now to the $(0,2)$-tensor field $\Phi$ on $(M, g)$ defined by $(1.1)$ and its associated endomorphism field $\varphi$. Instead of evaluating the vector field $\nabla^{*} \varphi$, we calculate the divergence of $\Phi$. Note that by (2.3) $\Phi$ is a symmetric tensor and thus $\operatorname{div} \Phi$ a 
one-form given as follows (see $\left[1\right.$, p.34]). Let $U_{1}, \cdots, U_{p}$ and $X_{1}, \cdots, X_{q}$ be (local) orthonormal frames of $L$ and $L^{\perp}$. Then (up to a for our purpose irrelevant conventional sign)

$$
(\operatorname{div} \Phi)(E)=\sum_{i=1}^{p}\left(\nabla_{U_{i}} \Phi\right)\left(U_{i}, E\right)+\sum_{\alpha=1}^{q}\left(\nabla_{X_{\alpha}} \Phi\right)\left(X_{\alpha}, E\right)
$$

But

$$
\Phi(E, F)=\kappa\left(T_{E} F\right)=g\left(T_{E} F, \tau\right)
$$

so that

$$
\begin{aligned}
\left(\nabla_{U_{i}} \Phi\right)\left(U_{i}, E\right) & =g\left(\left(\nabla_{U_{i}} T\right)_{U_{i}} E, \tau\right)+g\left(T_{U_{i}} E, \nabla_{U_{i}} \tau\right) \\
\left(\nabla_{X_{\alpha}} \Phi\right)\left(X_{\alpha}, E\right) & =g\left(\left(\nabla_{X_{\alpha}} T\right)_{X_{\alpha}} E, \tau\right)+g\left(T_{X_{\alpha}} E, \nabla_{X_{\alpha}} \tau\right)
\end{aligned}
$$

Next, we use the formulas of Gray [6] for $T$ and the integrability tensor $A$ ( $\mathrm{O}$ in Gray's notation) given by

$$
A_{E} F=\pi^{\perp}\left(\nabla_{\pi E} \pi F\right)+\pi\left(\nabla_{\pi E} \pi^{\perp} F\right)
$$

(O'Neill's formula apparatus for the tensors $T$ and $A$ is developed in [10] for the special context of Riemannian submersions only, while we need here Gray's more general context.) Then for $E=Y \in \Gamma L^{\perp}$ we have by (2.3) that $T_{X_{j}} Y=0$, and by $[6,(2.5)]$

$$
\left(\nabla_{X_{\alpha}} T\right)_{X_{\alpha}} Y=-T_{A_{X_{\alpha}} X_{\alpha}} Y=-T_{\pi^{\perp}\left(\nabla_{X_{\alpha}} x_{\alpha}\right)} Y
$$

which by $(2.3 \mathrm{~b})$ is a vertical vector field. It follows that

$$
g\left(\left(\nabla_{X_{\alpha}} T\right)_{X_{\alpha}} Y, \tau\right)=0
$$

Moreover, by $[6,(2.4)]$

$$
g\left(\left(\nabla_{U_{i}} T\right)_{U_{i}} Y, \tau\right)=-g\left(\left(\nabla_{U_{i}} T\right)_{U_{i}} \tau, Y\right)=-g\left(\pi\left(\left(\nabla_{U_{i}} T\right)_{U_{i}} \tau\right), Y\right)
$$

But by $[6,(2.9)]$ we have

$$
\pi\left(\left(\nabla_{U_{i}} T\right)_{U_{i}} \tau\right)=0
$$

so that

$$
g\left(\left(\nabla_{U_{i}} T\right)_{U_{i}} Y, \tau\right)=0
$$


It follows that for $Y \in \Gamma L^{\perp}$

$$
(\operatorname{div} \Phi)(Y)=\sum_{i=1}^{p} g\left(T_{U_{i}} Y, \nabla_{U_{i}} \tau\right)
$$

Note that $T_{U_{i}} Y$ is vertical by $(2.3 \mathrm{~b})$, so that

$$
(\operatorname{div} \Phi)(Y)=\sum_{i=1}^{p} g\left(T_{U_{i}} Y, \pi^{\perp} \nabla_{U_{i}} \tau\right)=\sum_{i=1}^{p} g\left(T_{U_{i}} Y, T_{U} \tau\right)
$$

Applied to the horizontal mean curvature vector field $\tau$ itself, we find the expression

$$
(\operatorname{div} \Phi)(\tau)=\sum_{i=1}^{p} g\left(T_{U_{i}} \tau, T_{U_{i}} \tau\right)=\sum_{i=1}^{p}\left|\pi^{\perp}\left(\nabla_{U_{i}} \tau\right)\right|^{2}
$$

From this, the result in the theorem is now clear. Obviously $\tau=0$ implies the vanishing of $\operatorname{div} \Phi$ and hence $\nabla^{*} \varphi=0$. Conversely, the vanishing of $\operatorname{div} \Phi$ implies $T_{U_{i}} \tau=0$ for $i=1, \cdots, p$. It follows that

$$
g\left(T_{U_{i}} \tau, U_{i}\right)=-g\left(\tau, T_{U_{i}} U_{i}\right)=0
$$

Then we see by means of (2.4) that $\operatorname{div} \Phi=0$ implies $g(\tau, \tau)=0$ and hence $\tau=0$.

Note that this calculation also shows that in the case of vanishing $\tau$ the tensor $\Phi$ itself vanishes according to definition (1.1). Thus $\operatorname{div} \Phi=0$ implies $\Phi=0$, and then the associated endomorphism $\varphi$ reduces to the canonical projection $T M \rightarrow M$.

REMARK. For the case $p=1$ the harmonicity of $\mathcal{F}$ means that all leaves are totally geodesic. For the case of codimension $q=1$ it is of interest to compare (3.8) with the divergence formulas in [14, p.92]. Thus, let $Z$ be the unit normal vector field to the (oriented and transversally oriented) foliation $\mathcal{F}$. Then by $[14,(7.34)$ and $(7.36)]$

$$
\operatorname{div} Z=-g(\tau, Z)
$$

and the vanishing of this expression characterises the harmonicity of $\mathcal{F}$. The divergence formula (3.8), valid in arbitrary codimension, and whose vanishing again characterises harmonicity, in contrast involves covariant derivatives of $\tau$ along the leaves.

\section{REFERENCES}

[1] A.L. Besse, Einstein manifolds, Ergeb. Math. Grenzgeb. 3. Folge 10 (Springer-Verlag, Berlin, Heidelberg, New York, 1987). 
[2] J. Eells and L. Lemaire, 'A report on harmonic maps', Bull. London Math. Soc. 10 (1978), 1-68.

[3] J. Eells and L. Lemaire, 'Another report on harmonic maps', Bull. London Math. Soc. 20 (1988), 385-524.

[4] J. Eells and J.H. Sampson, 'Harmonic mappings of R.iemannian manifolds', Amer. J. Math. 86 (1964), 109-160.

[5] E. García-Río, L. Vanhecke and M.E. Vázquez-Abal, 'Harmonic endomorphism fields', Illinois $J$. Math. (to appear).

[6] A. Gray, 'Pseudo-Riemannian almost product manifolds and submersions', J. Math. Mech. 16 (1967), 715-737.

[7] F. Kamber and Ph. Tondeur, 'Harmonic foliations', Lecture Notes in Math. 949 (1982), 87-121.

[8] F. Kamber and Ph. Tondeur, 'Foliations and metrics', Progr. Math. 32 (1983), 103-152.

[9] X. Masa, 'Duality and minimality in Riemannian foliations', Comment. Math. Helv. 67 (1992), 17-27.

[10] B. O'Neill, 'The fundamental equations of a submersion', Michigan Math. J. 13 (1966), 459-469.

[11] B.L. Reinhart, Differential geometry of foliations, Ergeb. Math. Grenzgeb. 99 (SpringerVerlag, Berlin, Heidelberg, New York, 1983).

[12] R. Rummler, 'Quelques notions simples en géométrie riemannienne et leurs applications aux feuilletages compacts', Comment. Math. Helv. 54 (1979), 224-239.

[13] D. Sullivan, 'A homological characterization of foliations consisting of minimal surfaces', Comment. Math. Helv. 54 (1979), 218-223.

[14] Ph. Tondeur, Foliations on Riemannian manifolds, Universitext (Springer-Verlag, Berlin, Heidelberg, New York, 1988).

[15] Ph. Tondeur, 'Geometry of Riemannian foliations', in Seminar on Math. Sciences 20 (Keio University, Yokohama, 1994).

[16] Ph. Tondeur, 'Riemannian foliations and tautness', Proc. Sympos. Pure Math. 54 (1993), 667-672.

[17] K. Yano and S. Ishihara, Tangent and cotangent bundles, Pure and Appl. Math. 16 (Marcel Dekker, New York, 1973).

Department of Mathematics

University of Illinois at Urbana-Champaign

1409 West Green Street

Urbana, Il 61801

United States of America
Department of Mathematics

Katholieke Universiteit Leuven

Celestijnenlaan 200B

B-3001 Leuven

Belgium 